\title{
Arthur Bispo do Rosário: prendre vie dans une métaphore délirante
}

\section{Arthur Bispo do Rosário: coming alive in a delirious metaphor}

\author{
Silvia Lippi*1 \\ Patrice Diaz ${ }^{* 2}$ \\ Patrick de Neuter*3
}

378

Les auteurs analysent l'articulation entre délire et création chez l'artiste plasticien Arthur Bispo Do Rosario. Interné à l'hôpital psychiatrique lorsqu'il était dans la trentaine, c'est à l'asile que Bispo crée son ouvre. Quelle relation existe-t-il entre le discours délirant et la production de l'objet (d'art)? Et quelle place tient l'œuvre pour le sujet? Vient-elle remplacer le délire, ou alors, lui donner consistance?

Mots clés: Délire, création, holophrase, objet

*1 Université Diderot - Paris 7 (Paris, France).

*2 Etablissement Public de Santé Barthélémy Durand (Étampes, France).

*3 Université Catholique de Louvain (Louvain-la-Neuve, Belgique). 
La psychanalyse s'intéresse, depuis toujours, à la création artistique et à sa relation avec l'inconscient. Freud avec le concept de sublimation, et Lacan à travers celui de sinthome, ${ }^{1}$ se sont questionnés sur la vie de certains artistes et sur la spécificité de leur œuvre.

Notre propos ne sera pas celui de faire de la psychanalyse appliquée à l'art, mais de nous laisser instruire par son langage (Maniglier, 2010). Il ne s'agira pas d'expliciter, à partir des concepts psychanalytiques, ce qui est implicite dans l'œuvre du plasticien Arthur Bispo do Rosario, mais de penser l'articulation entre délire et création et leurs fonctions spécifiques pour le sujet. Quelle relation y a-t-il entre le discours délirant et la production d'un objet (ici un objet d'art)? Et quelle place tient l'œuvre dans la vie du sujet? Vient-elle remplacer le délire, ou alors, lui donner consistance?

\section{Introduction}

«Un jour je suis simplement apparu sur terre » (Laude, 2013, p. 3): c'est ainsi que Arthur Bispo do Rosario résumait ses origines. L'artiste est né à Japartuba, une région pauvre du Brésil, en 1909 ou 1911. Il est mort à Rio de Janeiro en 1989. Ses parents, descendants d'esclaves noirs, travaillaient dans une exploitation de canne à sucre. A l'âge de quinze ans, il s'engage dans la marine militaire où il devient fusilier. En tant que boxeur, il participe à

${ }^{1}$ Dans la théorie lacanienne, un sinthome est un symptôme au sens élargit du terme, qui permet le nouage entre le réel, l'imaginaire et le symbolique. Il se forme indistinctement dans la névrose comme dans la psychose, mais selon des modalités différentes (Lacan, 1975-1976/2005). 
plusieurs championnats en Amérique latine. Il quitte l'armée à l'âge de vingtdeux ans, après avoir été condamné plusieurs fois pour insubordination.

On le retrouve à Rio de Janeiro où il exerce des petits métiers en échange du logement ou de nourriture : il est gardien, il est portier domestique.

Le 22 décembre 1938 il a une vision: le Christ lui apparait escorté de sept anges auréolés de bleu. Il erre dans les rues de Rio avant d'arriver au monastère de Sao Bento, où il déclare avoir été envoyé par Dieu: il est investi d'une mission réparatrice et salvatrice pour l'humanité entière. Les moines l'amènent à l'Hôpital psychiatrique.

L'année suivante il a des nouvelles visions: les anges lui ordonnent d'offrir à Dieu une représentation du monde le jour de son passage dans l'audelà. Sous le diagnostic de schizophrénie paranoïde, Bispo est définitivement interné à la Colonie Juliano Moreira, hôpital psychiatrique de la banlieue de Rio de Janeiro, où il séjournera pendant cinquante ans, jusqu'à sa mort.

C'est à l'asile que Bispo crée son œuvre. Plus de mille pièces. Il accumule les objets collectés dans l'hôpital en fouillant les poubelles et en récupérant tout ce qu'il trouve ou ce que les gardiens veulent bien lui donner: carton, morceaux de bois, jouets, petit mobilier, ustensiles de cuisine, vêtements, chaussures, pantoufles, bottes, tongs, chaussettes, peignes, chapeaux, fourchettes, bouteilles en plastiques, tissus...

Avec les déchets de l'hôpital, Bispo va recréer le monde. Pour présenter à Dieu ce monde, il confectionnera le Manteau de la présentation (Fig. I), réalisée à partir d'une couverture et de différentes étoffes colorées.

C'est après sa mort que l'œuvre de Bispo sera reconnue internationalement. ${ }^{2}$ Le plasticien ne s'est jamais considéré lui-même comme un artiste. Il était en revanche très concerné par sa fonction salvatrice, qui lui avait été accordée par Dieu. Autrement dit, par son délire. Nous verrons que dans son cas, la création artistique, plutôt que de le protéger du délire, ${ }^{3}$ se met à son service et l'« exploite »; pour Bispo, l'un ne va pas sans l'autre.

${ }^{2}$ L'œuvre de Bispo est révélée à la critique internationale en 1995 lors de la XXXXVI ${ }^{\text {ème }}$ Biennale de Venise (Arteaga, 2003, pp. 81-103). Elle est remarquée tout d'abord par Nise da Silveira, le psychiatre de l'asile d'orientation jungienne, et par la suite, par le critique d'art Frederico Morais, qui la présentera au public en 1982, au Musée d'Art Moderne de Rio de Janeiro. Rappelons aussi la présence de la psychologue Rosangela Maria Magalhaes, qui résida à la Colonie de 1981 à 1982 et qui a pu soutenir Bispo dans son travail artistique, devenant une sorte de muse pour lui.

${ }^{3}$ C'est le cas de Joyce, selon l'hypothèse de Lacan (1975-1976/2005). 


\section{LITERATURA, ARTE, PSICOPATOLOGIA}

Fig. 1 - Manteau de la présentation

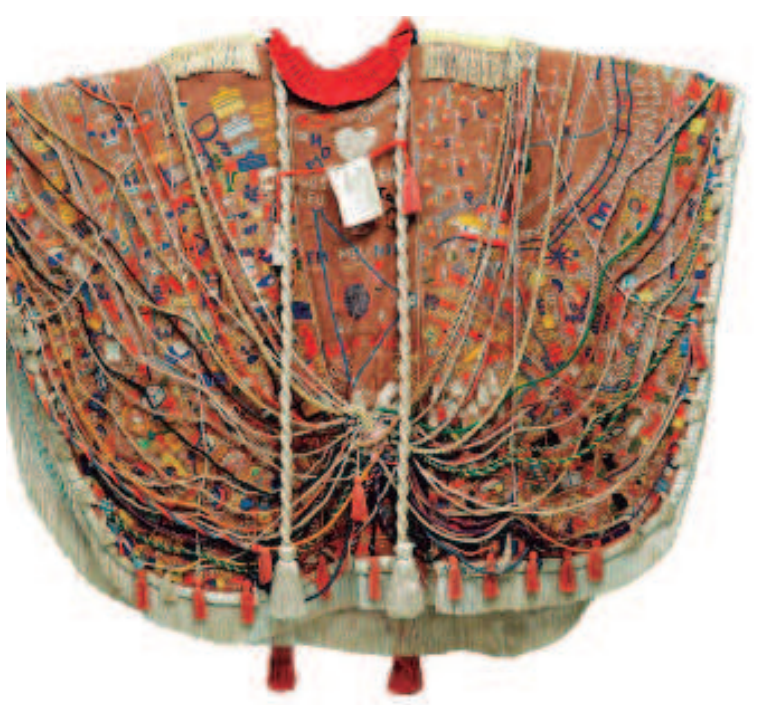

Chez certains artistes, le délire est à l'origine de l'acte créateur et ne s'estompe pas avec celui-ci. ${ }^{4}$ A propos du délire de Bispo, l'ethnologue J.-Y. Laude écrit: «Dieu insinua dans son esprit de Noir, pauvre, rejeté, mais choisi, que le monde avait pris une tournure erronée. A lui, Bispo de le réorienter. Il obéit. Il commença par réorganiser son propre environnement, celui de la colonie, avec l'idée de fonder un royaume dont il serait le roi et dans lequel, selon ses dires, il n'y aurait ni abîme ni tristesse» (Maniglier, 2010, p. 23). Il est important de souligner, à la suite de $\mathrm{M}$. Baudson, que «C'est au moment de son internement à la Colonie Juliano Moreira que son processus créatif, soutenu par ses délires mystiques, se met en marche» (Baudson, 2003, p. 126). Le délire donne à Bispo une «renaissance» qui se transforme en «acte», tout d'abord avec la création artistique, et par la suite, à travers la circulation du nom de l'artiste dans la communauté: d'abord dans la communauté psychiatrique $^{5}$ de la Colonie Juliano Moreira, et après, dans le monde des arts plastiques. Néanmoins, Bispo ne quittera jamais l'asile. En ce sens, l'acte créatif, pour Bispo, n'a pas la même fonction que l'écriture pour Joyce, qui empêchera à l'écrivain l'entrée dans la psychose.

${ }^{4}$ L'écrivain de science-fiction Philip Dick affirme écrire à partir de son délire. Il en parle ouvertement dans une conférence en 1977 (Dick Ph., 1998, pp. 154-155).

${ }^{5}$ Bispo ne connut pas d'entraves à son chantier, au contraire, il reçut appuis et faveurs des différents psychiatres, gardiens et patients de l'institution. Tout un réseau s'était organisé autour de lui pour lui fournir des nouveaux rebuts. 
L'analyse de certains éléments de l'œuvre de Bispo nous permettra d'approfondir le lien entre délire, création et acte subjectif. Comme se le demande J.-Y. Laude, à propos de Bispo: «Ne prouvait-il pas, à lui tout seul, le bien-fondé de l'art-thérapie, alternative paisible et possible à la réduction chimique des expressions agressives de la démence?» (Maniglier, 2010, p. 24).

\section{L'œuvre de Bispo: de l'accumulation à la reconstruction}

La production artistique de Bispo ne peut pas être considérée exclusivement comme une forme d' "art brut», si on entend par ce terme un art spontané, sans prétentions culturelles et qui ne suit aucune démarche intellectuelle. S'il est vrai que Bispo était un autodidacte qui travaillait en tant qu'artiste à l'hôpital psychiatrique, sa démarche créative reste très construite. Précisons que Bispo n'a jamais fréquenté les ateliers d'art-thérapie de l'hôpital: «Il a su échapper [à la fois] à l'artisanat thérapeutique et aux règles traditionnelles de l'art moderne» écrit R. Aquino, le directeur du Musée Bispo do Rosario à Rio de Janeiro. Néanmoins, continue-t-il, «Son œuvre, créée à l'écart des institutions et demeurant à l'écart du système commercial du marché de l'art, souffre d'avoir été classée comme un art mineur, avec des préjugés qui se retrouvent dans des dénominations telles que art psychologique ou art psychopathologique» (Aquino, 2003, p. 10).

L'ensemble des œuvres de Bispo est marqué par une répétition thématique compulsive fixée à des éléments du passé. Les allusions sont fréquentes à la marine (signaux, bateaux) et aux noms de pays. Les éléments de l'environnement physique sont regroupés en fonction des activités liées au sport, à la construction, au transport, à la campagne et au loisir. Son vocabulaire «plastique» tourne autour des mêmes éléments - les nations, les moyens de transport, les bateaux, les signaux de communication, les drapeaux, les sports, la construction - qui se combinent sous forme de récits (écritures) ou d'agglomérations d'objets.

Bispo compose un labyrinthe dans lequel des histoires sont parcourues, mais il est difficile de trouver le fil qui permet de sortir de ce labyrinthe: la logique inconsciente de l'artiste nous échappe.

Dans beaucoup de ses œuvres, et en particulier dans la série des étendards, nous remarquons une suite interminable de mots inscrits sur des lignes et séparés par des barres de la hauteur de l'interligne qui permettent de détacher un mot de l'autre (Étendard: je précise les mots qui sont écrits, 


\section{LITERATURA, ARTE, PSICOPATOLOGIA}

Fig. II). Il y a souvent une figure centrale autour de laquelle se répartissent d'autres éléments périphériques disposés selon une liberté absolue. Mais ses compositions hétéroclites ne sont pas le fruit de l'arbitraire: elles sont «reliées entre elles par le fil d'une cohérence intime gravée dans le registre mental de l'auteur» (Maniglier, 2010, p. 24) bien que cette cohérence ne suive aucun ordre ou planification.

Fig. 2 - Étendard : je précise les mots qui sont écrits

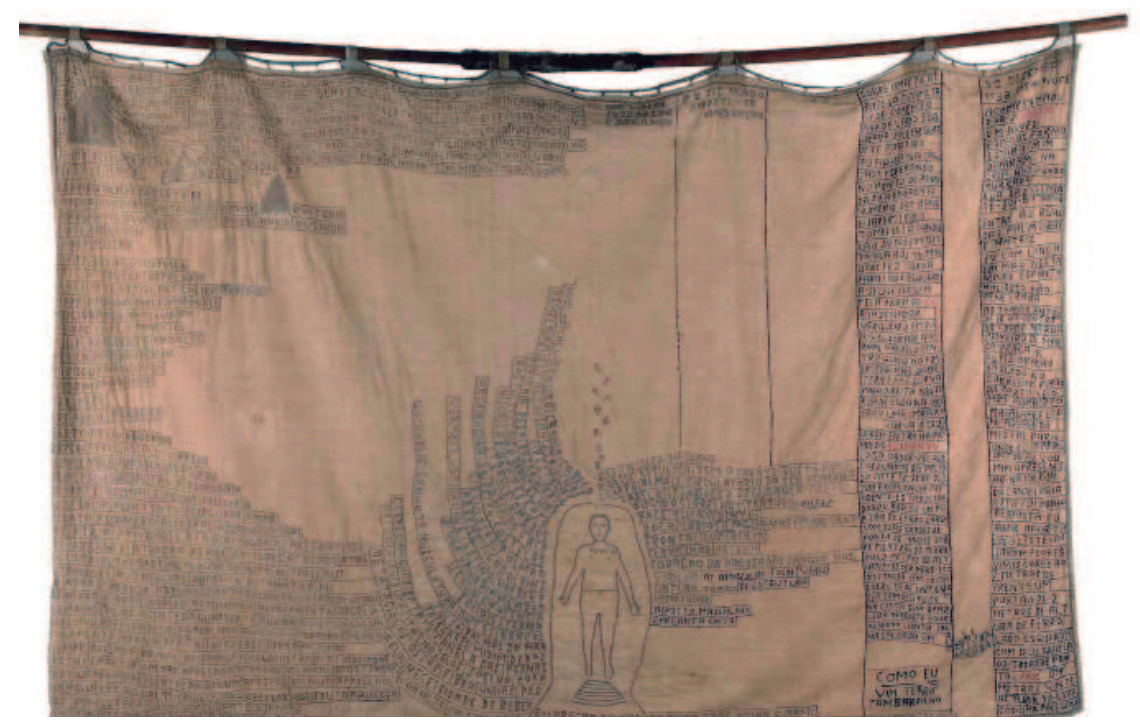

Lorsque, de l'autre côté de l'Atlantique, Duchamp détournait une roue de vélo pour la transformer en œuvre d'art, Bispo en dressait La roue de la fortune (Fig. III). Mais il n'avait aucune intention de s'inscrire dans le courant dadaïste: la roue de Bispo reste au-delà de l'intellectualisation et de la lutte contre les conventions idéologiques, esthétiques et politiques du mouvement que Bispo, reclus à l'hôpital psychiatrique, ne connaissait pas. Et pendant que Arman exposait des accumulations d'objets dans des bocaux de toutes formes, Bispo additionnait ses ustensiles au mépris des modes et en se fiant à sa seule rigueur, dans le but de réaliser son «projet» psychique et plastique, à partir de son délire. La rigueur du délire psychotique ${ }^{6} \mathrm{~s}^{\prime}$ applique, cette fois-ci, à la discipline artistique.

${ }^{6}$ « La psychose est un essai de rigueur. » (Lacan, 1975). 
Fig. 3 - La roue de la fortune

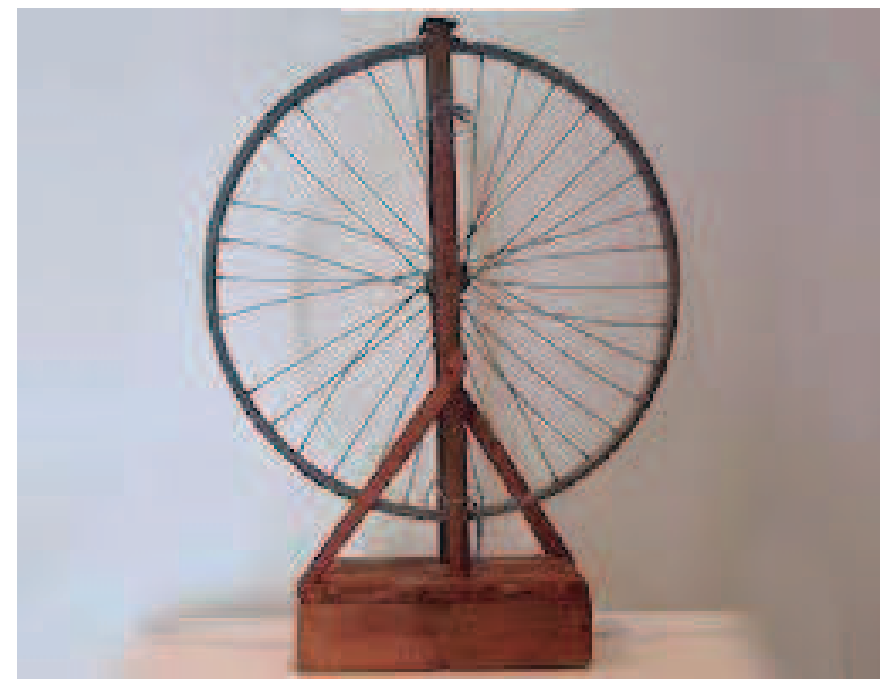

L'univers de Bispo nous rappelle celui du peintre italien Giorgio Morandi (1890-1964) qui a représenté tout au long de sa vie les mêmes objets (surtout des bouteilles) faisant partie de son petit univers, et objets qu'il accumulait dans son atelier de Bologne (Nature morte, Fig. IV). Comme Bispo, Morandi était isolé, ${ }^{7}$ indifférent aux courants artistiques, totalement concentré sur ses objets quotidiens qui reviennent dans chaque tableau selon des combinaisons infinies. Pour les deux artistes, les objets prennent une valeur presque sacrée, d'autant plus pour Bispo, pour qui les objets de ses compositions - à l'origine des rebuts récoltés à l'hôpital — sont tous directement ou indirectement adressés à Dieu.

Lacan met en évidence la propension à la production d'écrits, dessins et œuvres de tout genre dans les psychoses hallucinatoires ou délirantes. Il souligne la dimension «objectale» (Lacan, 1955-1956/1981, p. 89) de cette production, en raison du statut souvent ordinaire de l'objet, et du fait que la qualité artistique n'est pas toujours assurée. Pour Bispo, c'est l'inverse: l'objectale se transforme en «sacrée», l'objet-déchet devient «objet vénérable», digne d'attention, soigné, travaillé. Autrement dit, l'objet est élevé à la dignité de la Chose, ${ }^{8}$ pour reprendre la définition lacanienne de la sublimation.

${ }^{7}$ Soulignons que Morandi n'a jamais eu de relation avec la psychiatrie.

${ }^{8}$ Lacan reprend à Freud le concept de la Chose pour désigner l'objet inaccessible, l'impossible, à jamais perdu (Lacan, 1959-1960/1986, p. 169). 


\section{LITERATURA, ARTE, PSICOPATOLOGIA}

Fig. 4 - Nature morte

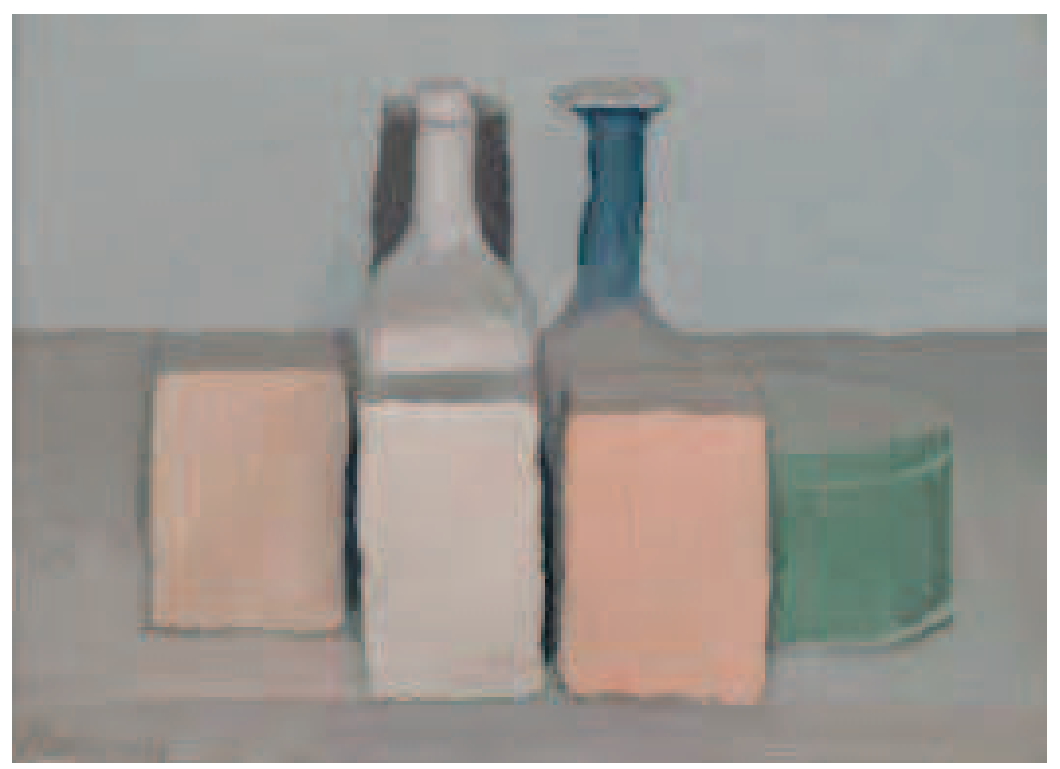

La tension avec le Réel ${ }^{9}$ est en jeu dans l'œuvre de Bispo, sans que l'aspect artisanal soit renié: son travail de broderie est stupéfiant (Baudson, 2003, p. 126). La communication est un des ses thèmes préférés, qu'il représente métaphoriquement à travers des symboles (Laude, 2013, p. 99): antennes de télévision, câbles électriques, téléphoniques et télégraphiques, moyens de transport comme les automobiles, les avions, les bateaux, les trains et autres, qui ont aussi la fonction de relier les éléments de ses compositions lorsque la structure est désordonnée ou dénouée de cadre. Représenter la communication est une tentative de partager son monde avec les autres. ${ }^{10}$

${ }^{9}$ Chez Lacan, le Réel n'est pas synonyme de «réalité» (effet de l'imaginaire fantasmatique), ni de «réalité psychique» au sens freudien du terme (rêve, fantasme etc.), réalité psychique qui est sujette à interprétation. Par Réel nous entendons ici ce qui échappe à toute tentative d'imaginarisation et/ou de symbolisation.

${ }^{10} \mathrm{C}$ 'est encore Dick qui souligne le problème de l'incommunicabilité pour le sujet psychotique: «Le problème c'est que si les mondes subjectifs sont vécus aussi différemment, la communication est interrompue... et c'est là que se situe la vraie maladie.» (Dick, Ph, 1998, p. 187). D'après le philosophe Bertrand Russel, «Le fou qui se prend pour un œuf poché ne peut être condamné que parce qu'il appartient à une minorité» (Russel, 2002, p. 22). 
Bispo découd son linge afin de broder une stupéfiante logorrhée verbale : récits, recensements, sermons... C'est de cette façon qu'il arrive à s'exprimer, à travers ses inversions de mots, ses néologismes et ses audaces de vocabulaire, qu'on retrouve dans ses œuvres plastiques. Mélange - confusion ? entre image, corps, objet, parole, communication, histoire: tous ces éléments sont présents dans son œuvre.

\section{Un assemblage d'objets-signifiants pour réorganiser le langage}

Lacan remarque «l'extériorité du psychotique par rapport à l'ensemble de l'appareil du langage». Il arrive même à se poser la question «de savoir si le psychotique est vraiment entré dans le langage» (Lacan, 1955-1956/1981, p. 284). Lacan part de l'hypothèse que «Si le névrosé habite le langage, le psychotique est lui habité, possédé, par le langage.» Prenons comme exemple l'automatisme mental. Dans l'hallucination verbale, le sujet parle tout seul, à haute voix. Quelque chose en dehors du sujet parle à son insu: il est parlé plus qu'il ne parle, autrement dit, c'est l'Autre ${ }^{11}$ qui parle à sa place. ${ }^{12}$

«[...] le psychotique est un martyr de l'inconscient» soutient Lacan, un martyr qu'il considère aussi comme un «témoin» : «[...] Le psychotique $[\ldots]$ semble fixé, immobilisé dans une position qui le met hors d'état de restaurer authentiquement le sens de ce dont il témoigne, et de le partager dans le discours des autres.» (Lacan, 1955-1956/1981, p. 149).

La forclusion du Nom-du-Père, conséquence de l'échec de la métaphore paternelle, provoque, aux yeux de Lacan, une carence de la «signification phallique» ${ }^{13}$ (Lacan, 1957/1966, p. 558), en raison de l'absence du signifiant qui vient donner du sens rétroactivement à un autre signifiant. Cela empêche le sujet d'être dans un discours partageable. En d'autres termes, un sens ne vient pas stabiliser la phrase, qui ne peut donc aboutir. Les troubles du

${ }^{11}$ Par Autre, nous désignons en général, l'ordre symbolique qui détermine le sujet. Dans la psychose, l'Autre vient indiquer toute extériorité menaçante et inquiétante pour le sujet.

${ }^{12}$ C'est ce que Gaétan De Clérambault appelle «automatisme majeur» (De Clérambault, 1992).

${ }^{13}$ La signification phallique se produit, grâce à la métaphore paternelle, lorsque les phrases sont capitonnées par le sens et pilotés par le fantasme. 


\section{LITERATURA, ARTE, PSICOPATOLOGIA}

langage (diffluence, discordance, barrage, néologisme, dysphasie, dyslexie...) sont l'un des effets possibles du dérèglement de la chaîne symbolique dans la psychose.

Rappelons que $\mathrm{S}_{1}$ est, dans la chaine des signifiants, le trait identificatoire qui permet à un sujet d'advenir. Il fonctionne comme support de la différence et, ne pouvant pas se signifier lui-même, fait appel à un second signifiant, le signifiant binaire $\mathrm{S}_{2}$. Ce processus est en panne dans la psychose : les signifiants peuvent alors se superposer, comme s'il n'y avait plus d'espace entre les mots. Ce phénomène, appelé par Lacan «holophrase» (Lacan, 1964/1973, p. 215), bloque l'articulation entre les signifiants et contribue au dérèglement, voir la rupture, de la chaîne signifiante $\left(\mathrm{S}_{1}-\mathrm{S}_{2}\right)$.

L'holophrase présente une jouissance ${ }^{14}$ dans la parole indépendamment du message (Soler, 2009, p. 36). En ce sens, le discours du psychotique est encombré par l'Autre, à travers une jouissance qui le déborde. Le discours est adressé en tant que «témoignage» — témoignage d'un envahissement de jouissance — sans que le message soit transmissible.

Le délire est à la fois une forme de déchaînement de la jouissance, en même temps qu'une tentative de poser une limite à celle-ci: lorsqu'il arrive à se structurer, ${ }^{15}$ il peut restaurer le sens dans la phrase.

Le psychotique est bien sûr dans le langage, mais la structure de celui-ci est différente: dans la psychose, sa visée est plus jouissive que communicative. Le langage est incapable de donner au sujet une assise stable et une inscription permanente dans le lien social. Rappelons ce qui écrit Lacan en 1972: «[...] le schizophrène se spécifie d'être pris sans le secours d'aucun discours établi» ${ }^{16}$ (Lacan, 1972/2001, p. 474).

${ }^{14}$ Ici le terme de «jouissance» désigne une étrange satisfaction au-delà du principe de plaisir que Freud a découverte dans une série d'expériences de douleurs physiques ou psychiques. Lacan considère les expériences liées à la douleur et à l'excès, toujours immaitrisables, comme étant de l'ordre de la jouissance. Le symptôme en est un exemple.

${ }^{15}$ Nous distinguons le délire de la confusion mentale. A remarquer qu'en anglais delusion et en allemand Wahn signifient «délire», tandis que Delirium, dans les deux langues, désigne un état plus général de «confusion mentale».

${ }^{16}$ Lacan se réfère aux quatre discours dont il donne la structure, le discours du maître, le discours de l'université, le discours de l'hystérique, le discours de l'analyste. Les discours sont des «modes de traitement de la jouissance par le langage». (Askofaré et al., 2008, p. 573-587). 
Que se passe-t-il lorsque l'œuvre est mise au service du délire? Dans le cas de Bispo, l'acte créatif est favorisé par l'invention ${ }^{17}$ délirante, au point qu'il faut se demander si la production de l'artiste aurait été possible sans le délire. En ce sens, la question s'impose de savoir où situer l'acte du sujet: dans l'invention délirante ou dans l'œuvre?

Le délire se met en place dans la tentative de restaurer le sens. Le délire rassure le sujet à propos de son savoir: il transforme la croyance en certitude, certitude qu'il y a un Autre qui jouit à ses frais (Notons que la certitude est assumée seulement dans les délires systématisés, comme la paranoïa et la paraphrénie). La solidité du couple $\mathrm{S}_{1}-\mathrm{S}_{2}$ interdit toute ouverture dialectique dans le discours, l'holophrase fait émerger un savoir dépourvu d'ambiguïté, savoir dont le sujet ne doute pas, et qui peut même devenir une certitude partagée par les autres.

Cette certitude est absolument nécessaire à Bispo, elle lui donne la force de récolter les objets, de se faire aider par les autres, de les assembler dans les différentes compositions selon une logique précise, d'écrire, de dessiner, de broder... Et vice-versa, c'est l'acte créatif qui fait passer le délire de la croyance à la certitude: il y a un acte qui assure, à travers la production de l'objet (d'art), l'importance de la finalité de l'œuvre, œuvre qui sera offerte à Dieu le jour du jugement universel.

L'acte créatif est soutenu par le délire et le délire par l'acte créatif: l'un ne va pas sans l'autre, et c'est de cette façon que la suppléance ${ }^{18}$ se met en place, suppléance qui passe par la création de l'œuvre et par le délire paraphrénique. Pouvons-nous penser l'acte créatif comme une forme de paraphrénie? Et toute œuvre d'art, en tant que construction d'une «nouvelle réalité» (Freud, 1924/1973, p. 301), est-elle, dans son fond, délirante?

Chez Bispo, les déchets, comme des signifiants désarrimés, prennent forme grâce au délire. Mais l'acte créatif lui permet de réaffirmer la certitude d'une façon différente de celle du délire paranoïaque: comme si une démassification de $\mathrm{S}_{1}$ et $\mathrm{S}_{2}$ était en acte. Nous pouvons alors faire l'hypothèse qu'il existe un moment où le langage du psychotique se modifie, s'organise de

${ }^{17}$ «C'est précisément le traumatisme du signifiant, du signifiant énigme, du signifiant jouissance, qui oblige à une invention subjective, une invention du sens, toujours plus ou moins de l'ordre du délire » (Miller, 2004, p. 10).

18 «Suppléance comme substitution de ce qui manque sous le mode de la forclusion, dans la psychose » (Askofaré et al., 2011). 


\section{LITERATURA, ARTE, PSICOPATOLOGIA}

façon nouvelle, sans devenir pourtant un langage producteur d'un sens piloté par le fantasme comme celui du névrosé.

La paranoïa de Bispo se transforme en paraphrénie confabulante: en associant le délire à l'acte créatif, Bispo met en place son sinthome qui, en le faisant devenir une des figures dominantes des arts plastiques du XXème siècle, le fera aussi «tenir» loin des passages à l'acte et des décompensations autodestructrices.

\section{Le corps et le nom dans l'œuvre}

Pour Bispo, l'acte créatif vient s'articuler au discours (délirant) et à la jouissance, afin que celle-ci ne concoure plus à l'anéantissement du sujet. Si le délire peut apporter une limite à la jouissance, l'acte créatif opère à travers une réinscription de la jouissance, jouissance qui n'est plus traumatique et anéantissante pour le sujet. En tant que sinthomes, délire et création permettent de rétablir le nouage entre Réel, Symbolique et Imaginaire: c'est la question du nom propre et du corps qui sont alors convoqués.

La poiesis de Bispo se fait sur plusieurs plans: images, paroles, objets, espaces qui se combinent entre eux ou pas. Dans la série des étendards, les éléments apparaissent dans une disposition spatiale inopinée. Le rapport entre image et écriture s'intensifie, comme si corps et parole cherchaient un lien entre eux. Le lien s'opère à travers l'acte créatif du sujet, acte qui devient tel, à travers la circulation de l'œuvre qui porte son nom.

Dans l'étendard qui a pour titre Eu Preciso destas palabras, «J'ai besoin de ces mots écrits» (Fig. 2), une figure humaine occupe le centre de l'œuvre, et de ce personnage surgissent des descriptions sur le corps humain et ses fonctions: «tête, crâne, cuir, poux, épaules, veine du visage, côte, angine de poitrine, reins, abdomen, intestins...». En bas de l'œuvre, Bispo écrit «no peito traz e nome proprio» («dans la poitrine il a un aigle c'est nom propre»), et encore plus bas «nomes proprios Aracy Aracaju Arthur Algérie», où Aracaju est le lieu d'origine de Bispo et Arthur son nom.

Nous pouvons voir ici, pour Bispo, une tentative de prendre corps dans l'œuvre. De même, par l'assemblage des déchets, ne leur donne-t-il pas vie, et de ce fait ne se donne-t-il pas vie lui-même à travers son œuvre?

Néanmoins, la forclusion du Nom-du-Père entraine une difficulté à faire circuler le nom du sujet dans le social. Le psychotique arrive difficilement à faire «sien» le nom propre: comme si ses actes ne pouvaient pas être signés. 
Cette opération est extrêmement difficile pour le psychotique, en raison de la forclusion du Nom-du-Père. Au niveau du fantasme, le psychotique peut tuer le père, mais il n'arrive pas à symboliser son meurtre à travers le nom qui devrait être associé à son acte, acte qui est à la fois meurtrier et créateur.

Deux cas célèbres viennent éclaircir notre propos: celui de Van Gogh et celui de Séraphine de Senlis. Vincent ne signa jamais ses tableaux «Van Gogh» (Martin-Mattera, 2011, p. 429), comme s'il ne pouvait pas en assumer la paternité, et Séraphine décompensera au moment de la célébrité et de la reconnaissance par la communauté, au moment où elle "se sera fait un nom » (Jodeau-Belle, \& Maleval, 2011, p. 624). Le fantasme parricide, pour les deux artistes, ne peut pas se réaliser.

Dans le cas de Bispo, le nom propre est intégré à ses compositions. Il existe à l'intérieur de l'œuvre. Il est un élément qui se mélange aux autres, métamorphosé en animal, en aigle, ou couvert par d'autres noms propres: le Nom-du-Père a-t-il perdu sa spécificité et son efficacité? Il ne capitonne pas les autres signifiants, et il ne lui permet pas de s'inscrire dans le lien social. Paradoxalement, pour Bispo, l'acte créatif annule son nom, tout en donnant au sujet une nouvelle vie à l'intérieur de l'œuvre.

\section{Conclusions}

L'œuvre de Bispo se montre à nos yeux comme un labyrinthe. Le névrosé, dans un labyrinthe, trouve facilement la clé pour s'en sortir. Il comprend l'organisation, la logique, il repère l'entrée et la sortie. Mais pour le plasticien Bispo, cette clé est introuvable: il n'y a ni début, ni fin, on ne repère pas l'organisation sous-jacente de l'œuvre.

En proposant l'hypothèse du labyrinthe, nous pensons quand même qu'une certaine organisation existe, quoique illisible. Et c'est cette organisation qui permet une certaine régulation de la jouissance. Bispo est en position d'objet par rapport à l'Autre (ici Dieu). Il est objet de sa jouissance mais sa création - son œuvre - , lui permet de différer cette jouissance, qui serait autrement insupportable.

Bispo n'a en effet qu'un seul interlocuteur, Dieu. Rappelons-nous que son délire mystique et mégalomaniaque l'accompagnera tout au long de sa vie. L'acte créatif donne la main au délire qui l'inspire et le soutient. Autrement dit, le Réel insiste dans l'acte artistique comme dans les signifiants du délire. Et qu'en est-il de cette jouissance qui n'a pas pu se canaliser 


\section{LITERATURA, ARTE, PSICOPATOLOGIA}

entièrement, ni dans le processus de sublimation ni à travers la circulation l'œuvre dans le monde de l'art?

Il faut bien faire ici la distinction d'une part, entre l'artiste et l'œuvre d'une part, et, d'autre part, entre l'artiste et le sujet. En effet, si l'œuvre elle-même est partageable - le Réel qu'elle véhicule nous concerne tous -, le sujet psychotique, en raison de sa non-inscription de l'ordre symbolique capitonné par le Nom-du-Père, vit la problématique sociale de manière frontale. Comme nous l'avons vu, cette problématique est intégrée directement dans l'œuvre, en particulière la question de la communication, représentée à travers les différents moyens: antennes de télévision, câbles électriques, téléphoniques, télégraphiques, automobiles, avions, bateaux, trains....

Bispo est artiste pour ceux qui le reconnaissent en tant que tel, mais lui, il ne se vit pas comme tel. Lui, il est l'élu de Dieu. Son œuvre s'adresse exclusivement à Dieu: le sujet accomplit son acte — son art — non en son nom, mais en celui de Dieu. Il manque, chez le sujet, une adresse autre que celle qu'il destine à un Autre Absolu. Il manque un espace entre le sujet et l'œuvre: l'œuvre est à la fois corps et sujet.

Comment le sujet, peut-il se déloger de cette identification à l'objet de la jouissance de l'Autre? Il ne s'agit pas, pour Bispo, comme pour beaucoup de schizophrènes, d'une identification à l'objet déchet, mais d'une incarnation de l'objet élu, l'objet élu de Dieu. Ni l'acte de création ni l'œuvre en elle-même ne protégeront Bispo de la jouissance du signifiant immaitrisable, qui passe par le délire et le soutient.

\section{Références}

Aquino R. (2003). L'artiste Arthur Bispo do Rosario. In Arthur Bispo do Rosario, Catalogue de l'exposition de la Galerie nationale du Jeu de Paume. Paris, pp. 9-11.

Arteaga A. (2003). Arthur Bispo do Rosario: l'homme dans son labyrinthe. In Arthur Bispo do Rosario, Catalogue de l'exposition de la Galerie nationale du Jeu de Paume. Paris, pp. 81-103.

Askofaré, S., \& Combres, L. (2011). Symptômes et suppléances. Un essai de problématisation. Recherches en Psychanalyse [revue en ligne], 2011. URL: http:// recherchespsychanalyse.revues.org/3080: 13-20.

Askofaré, S., Gaspard, J.-L., Macary-Garipuy, P., \& Sauret, M.-J. (2008). Une Rev. Latinoam. Psicopat. Fund., São Paulo, 21(2), 378-394, jun. 2018 
physique du sens? Le problème de la formalisation en psychanalyse. Revista Latinoamericana de Psicopatologia Fundamental, 11(4), 573-587.

Baudson, M. (2003). Arthur Bispo do Rosario. In Arthur Bispo do Rosario, Catalogue de l'exposition de la Galerie nationale du Jeu de Paume. Paris, pp. 125-127.

De Clérambault, G. (1992). L’Automatisme mental. Paris, France: Empêcheurs de penser en rond. p. 22.

Dick Ph., K. (1998). Si ce monde vous déplait... et autres écrits. Paris, France: L'Eclat. pp. 154-155.

Freud, S. (1973). La perte de la réalité dans la névrose et dans la psychose. In Névrose, psychose et perversion (pp. 299-303). Paris, France: PUF. (Travail original publié dans 1924).

Jodeau-Belle, L., \& Maleval, J.-C. (2011). Le sacrifice fait à Dieu de Séraphine de Senlis. L'évolution psychiatrique. Schizophrénie, 76(4), 617-630.

Lacan, J. (1966). D'une question préliminaire à tout traitement possible de la psychose. In Ecrits (pp. 531-583). Paris, France: Seuil. (Travail original publié dans 1957).

Lacan, J. (1973). Le séminaire. Livre XI. Les quatre concepts fondamentaux de la psychanalyse. Paris, France: Seuil. (Travail original publié dans 1964).

Lacan, J. (1975). Yale University, Kanzer Seminar. Scilicet, (6/7), 7-31.

Lacan, J. (1981). Le séminaire. Livre III. Les Psychoses. Paris, France: Seuil. (Travail original publié dans 1955-56).

Lacan, J. (1986). Le séminaire. Livre VII. L'éthique de la psychanalyse. Paris, France: Seuil. (Travail original publié dans 1959-60).

Lacan, J. (2001). L'étourdit. In Autres écrits (pp.449-495). Paris: Seuil. (Travail original publié dans 1972).

Lacan, J. (2005). Le séminaire. Livre XXIII. Le sinthome. Paris, France: Seuil. (Travail original publié dans 1975-76).

Laude, J.-Y. (2013). Pépites brésiliennes. Paris, France: Actes Sud.

Maniglier, P. (2010). La Perspective du Diable. Figurations de l'espace et philosophie de la Renaissance à Rosemary's Baby. Villa Arson: Actes Sud.

Martin-Mattera, P. (2011). Sublimation ou sinthomation? Apports et réflexions cliniques sur la création dans la psychose. L'évolution psychiatrique. Ecriture, 76(3), 419-432.

Miller, J.-A. (2004). L'invention psychotique. Quarto. Le marché des symptômes, 80(81), 6-16.

Russel, B. (2002). Histoire de la philosophie occidentale. Paris, France: Gallimard.

Soler, C. (2009). Lacan, l’inconscient réinventé. Paris, France: PUF. 


\section{LITERATURA, ARTE, PSICOPATOLOGIA}

\section{Resumés}

(Arthur Bispo do Rosário: ganhar vida numa metáfora delirante)

Os autores analisam a articulação entre delírio e criação na obra do artista plástico Arthur Bispo do Rosário. Internado no hospital psiquiátrico quando estava na casa dos 30 anos, Bispo criou sua obra no hospício. Que relação existe entre o discurso delirante e a produção de um objeto (de arte)? Que lugar ocupa a obra para o sujeito? Será que ela vem substituir o delírio ou dar-lhe consistência?

Palabras-chave: Delírio, criação, holófrase, objeto

(Arthur Bispo do Rosário: coming alive in a delirious metaphor)

The authors analyze the correlation between delirium and creation in the life of Brazilian plastic artist Arthur Bispo do Rosario. He was consigned to a psychiatric hospital in his early thirties and in that asylum, Bispo created his entire work. What kind of relationship is there between a delirious discourse and the creation of an (art) object? And, what kind of role does the work of art play for the subject? Can it replace his delirium, or does it reinforce it?

Key words: Delirium, creation, holophrase, object

(Arthur Bispo do Rosário: ganar vida en una metáfora delirante)

En la obra del artista plástico Arthur Bispo do Rosario, los autores analizan la articulación entre el delirio y la creación. Fue internado en el hospital psiquiátrico, cuando estaba en sus treintas, y allí, crea su obra. ¿Cuál es la relación entre el discurso delirante y la producción de un objeto (artístico)? ¿Qué lugar ocupa la obra para el sujeto? ¿Será que viene a substituir el delirio o a darle consistencia?

Palabras clave: Delirio, creación, holophrase, objeto

(Arthur Bispo do Rosário: In einer delirierenden Metapher lebendig werden)

Die Autoren analysieren den Zusammenhang zwischen Wahn und Schöpfung im Leben des bildenden Künstlers Arthur Bispo do Rosario. Dieser wurde mit 30 Jahren in eine psychiatrische Klinik eingeliefert, in welcher er dann sein künstlerisches Lebenswerk erschuf. Was für eine Art von Beziehung besteht zwischen dem delirierenden Diskurs und der Schaffung von (Kunst-) Objekten? Und welchen Platz nimmt das Werk beim Subjekt ein? Ersetzt es den Wahn oder verstärkt es ihn?

Schlüsselwörter: Wahn, Schaffung, Holophrase, Objekt 
Citação/Citation: Lippi, S., Diaz, P. \& De Neuter, P. (2018, junho). Arthur Bispo do Rosário: prendre vie dans une métaphore délirante. Revista Latinoamericana de Psicopatologia Fundamental, 21(2), 378-394. http://dx.doi.org/10.1590/1415-4714.2018v21n2p378.10.

Editores do artigo/Editors: Vários

Recebido/Received: 1.10.2017/ 10.1.2017 Aceito/Accepted: 30.1.2018 / 1.30.2018

Copyright: (C) 2009 Associação Universitária de Pesquisa em Psicopatologia Fundamental/ University Association for Research in Fundamental Psychopathology. Este é um artigo de livre acesso, que permite uso irrestrito, distribuição e reprodução em qualquer meio, desde que o autor e a fonte sejam citados / This is an open-access article, which permits unrestricted use, distribution, and reproduction in any medium, provided the original authors and sources are credited.

Financiamento/Funding: Os autores declaram não terem sido financiados ou apoiados. The authors have no support or funding funded to report.

Conflito de interesses/Conflict of interest: Os autores declaram que não há conflito de interesses / The authors have no conflict of interest to declare.

\section{Silvia LipPI}

Psychanalyste, Docteur en psychologie, chercheur associé au CRPMS (EA 3522), Université Diderot-Paris 7, psychologue hospitalière titulaire EPS Barthélémy Durand.

Avenue du 8 mai 1945

91152 Etampes, France

slippi@club-internet.fr

\section{Patrice Diaz}

Psychanalyste, psychologue EPS Barthélémy Durand.

Avenue du 8 mai 1945

91152 Etampes, France

\section{Patrick De Neuter}

Psychanalyste, Professeur émérite, Université Catholique de Louvain.

Place de l'Université 1

1348 Louvain-la-Neuve, Belgique UK CB2 2QQ

This is an open-access article, which permits unrestricted use, distribution, the original authors and sources are credited. 